Metz-Göckel, Sigrid

\title{
Auf den Spuren der wissenschaftlichen Drop-Outs
}

Die Hochschule : Journal für Wissenschaft und Bildung 29 (2020) 1, S. 76-86

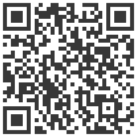

Quellenangabe/ Reference:

Metz-Göckel, Sigrid: Auf den Spuren der wissenschaftlichen Drop-Outs - In: Die Hochschule : Journal für Wissenschaft und Bildung 29 (2020) 1, S. 76-86 - URN: urn:nbn:de:0111-pedocs-238047 - DOI: 10.25656/01:23804

https://nbn-resolving.org/urn:nbn:de:0111-pedocs-238047

https://doi.org/10.25656/01:23804

in Kooperation mit / in cooperation with:

\section{Institut für Hochschulforschung (HoF)}

an der Martin-Luther-Universität Halle-Wittenberg

https://www.hof.uni-halle.de

\section{Nutzungsbedingungen}

Gewährt wird ein nicht exklusives, nicht übertragbares, persönliches und beschränktes Recht auf Nutzung dieses Dokuments. Dieses Dokument ist ausschließlich für den persönlichen, nicht-kommerziellen Gebrauch bestimmt. Die Nutzung stellt keine Übertragung des Eigentumsrechts an diesem Dokument dar und gilt vorbehaltlich der folgenden Einschränkungen: Auf sämtlichen Kopien dieses Dokuments müssen alle Urheberrechtshinweise und sonstigen Hinweise auf gesetzlichen Schutz beibehalten werden. Sie dürfen dieses Dokument nicht in irgendeiner Weise abändern, noch dürfen Sie dieses Dokument für öffentliche oder kommerzielle Zwecke vervielfältigen, öffentlich ausstellen, aufführen, vertreiben oder anderweitig nutzen.

Mit der Verwendung dieses Dokuments erkennen Sie die Nutzungsbedingungen an.

\section{Terms of use}

We grant a non-exclusive, non-transferable, individual and limited right to using this document.

This document is solely intended for your personal, non-commercial use. Use of this document does not include any transfer of property rights and it is conditional to the following limitations: All of the copies of this documents must retain all copyright information and other information regarding legal protection. You are not allowed to alter this document in any way, to copy it for public or commercial purposes, to exhibit the document in public, to perform, distribute or otherwise use the document in public.

By using this particular document, you accept the above-stated conditions of use.

\section{Kontakt / Contact:}

\section{peDOCS}

DIPF | Leibniz-Institut für Bildungsforschung und Bildungsinformation Informationszentrum (IZ) Bildung

E-Mail: pedocs@dipf.de

Internet: www.pedocs.de

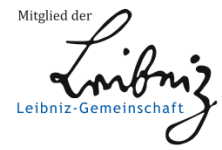




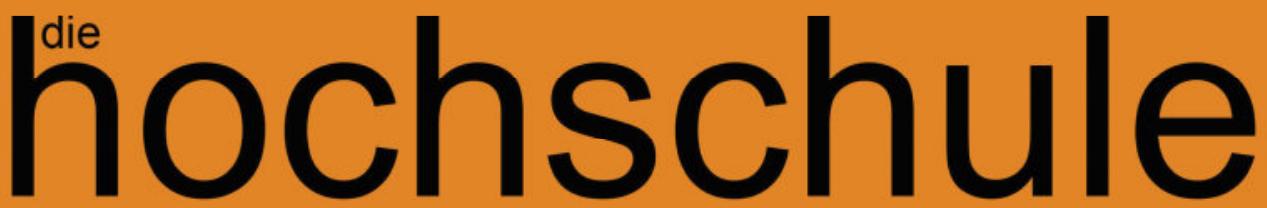

journal für wissenschaft und bildung

Sandra Beaufaÿs

Anja Franz

Svea Korff

(Hrsg.)

Ausstieg aus der Wissenschaft

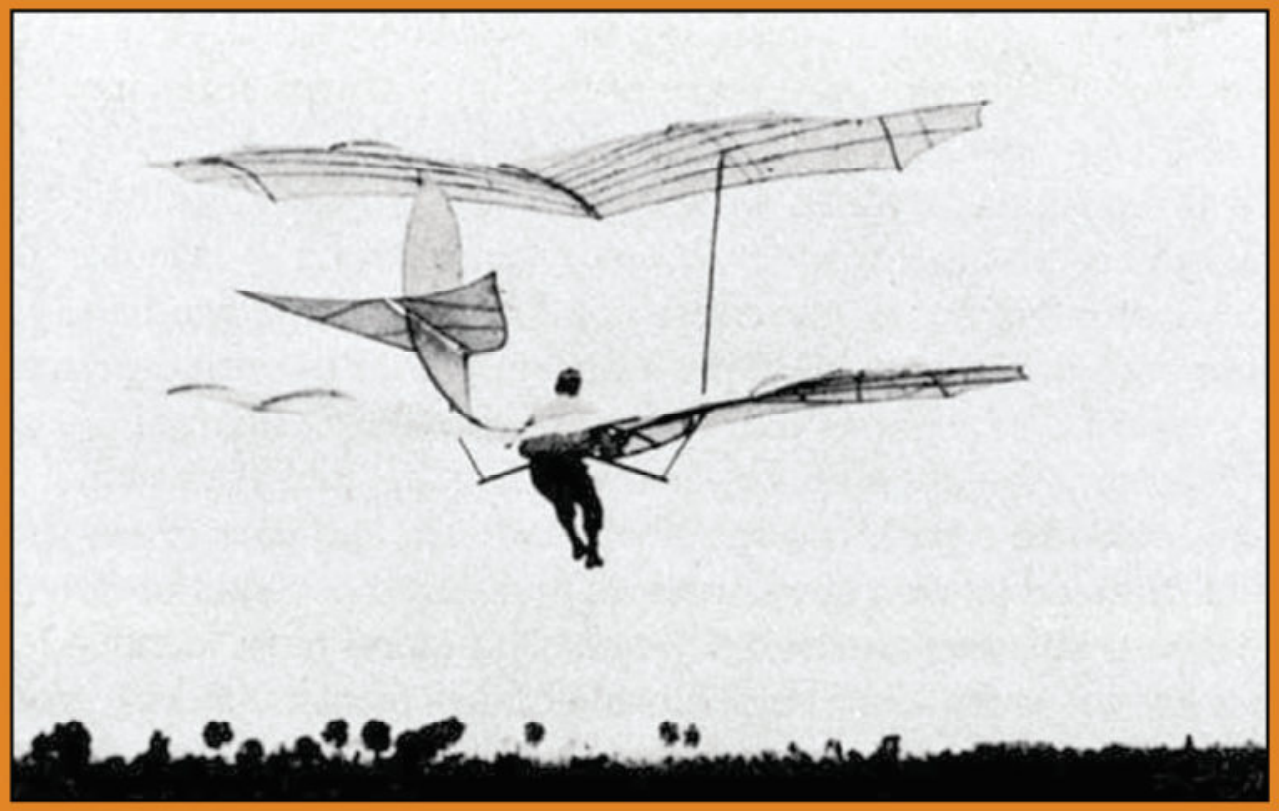




\title{
die hochschule. journal für wissenschaft und bildung
}

\author{
Herausgegeben von Peer Pasternack \\ für das Institut für Hochschulforschung (HoF) \\ an der Martin-Luther-Universität Halle-Wittenberg \\ Redaktion: Daniel Hechler
}
Institut für Hochschulforschung Halle-Wittenberg, Collegienstraße 62, D-06886 Wittenberg https://www.diehochschule.de
Kontakt Redaktion: daniel.hechler@hof.uni-halle.de

Konakt Vertrieb: Tel. 03491/466 254, Fax: 03491/466 255, eMail: institut@hof.uni-halle.de

ISSN 1618-9671, ISBN 978-3-937573-77-9

Die Zeitschrift „die hochschule“ versteht sich als Ort für Debatten aller Fragen der Hochschulforschung sowie angrenzender Themen aus der Wissenschafts- und Bildungsforschung. Als Beihefte der „hochschule“ erscheinen die "HoF-Handreichungen", die sich dem Transfer hochschulforscherischen Wissens vor allem in die Praxis der Hochschulentwicklung widmen.

Artikelmanuskripte werden elektronisch per eMail-Attachment erbeten. Ihr Umfang soll 25.000 Zeichen nicht überschreiten. Inhaltlich ist „die hochschule“ vorrangig an Beiträgen interessiert, die Themen jenseits des Mainstreams oder Mainstream-Themen in unorthodoxen Perspektiven behandeln. Eingereicht werden können sozialund geschichtswissenschaftliche Texte, die (a) auf empirischer Basis ein nachvollziehbar formuliertes Problem aufklären oder/und (b) eine theoretische Perspektive entfalten oder/und (c) zeitdiagnostisch angelegt sind, ohne reiner Meinungsartikel zu sein. Für Rezensionen beträgt der Maximalumfang 7.500 Zeichen. Weitere Autorenund Rezensionshinweise finden sich auf der Homepage der Zeitschrift: www.diehochschule.de $\gg$ Redaktion.

Das Institut für Hochschulforschung Halle-Wittenberg (HoF), 1996 gegründet, ist ein An-Institut der Martin-Luther-Universität (www.hof.uni-halle.de). Es hat seinen Sitz in der Stiftung Leucorea Wittenberg und wird geleitet von Peer Pasternack.

Als Beilage zu "die hochschule“ erscheint der „HoF-Berichterstatter“ mit aktuellen Nachrichten aus dem Institut für Hochschulforschung Halle-Wittenberg. Daneben publiziert das Institut die „HoF-Arbeitsberichte“ (https://www.hof.uni-halle.de/publikati onen/hof_arbeitsberichte.htm) und die Schriftenreihe „Hochschul- und Wissenschaftsforschung Halle-Wittenberg" beim BWV Berliner Wissenschafts-Verlag. Ein quartalsweise erscheinender eMail-Newsletter kann abonniert werden unter https://I ists.uni-halle.de/mailman/listinfo/hofnews

Abbildung vordere Umschlagseite: Otto Lilienthal am 19. Oktober 1895 mit seinem größeren Doppeldecker. Reproduktion durch Neuhauss/Fülleborn (https://commons.wikimedia. org/wiki/File:LilienthalDoppeldecker_1895-10-19.jpg) 


\section{INHALT}

\section{Ausstieg aus der Wissenschaft}

Sandra Beaufä̈s, Anja Franz, Svea Korff:

Ausstieg aus der Wissenschaft. Zur Einleitung.

Gesche Brandt, Anja Franz:

Promotionsabbrecher*innen in Deutschland. Stand der Forschung und

Perspektiven .16

\section{Barbara Hendriks:}

Der geplante Ausstieg aus der Wissenschaft. Ein Praxisbeispiel aus

strukturierten Promotionsformen der Geistes-, Kultur- und

Gesellschaftswissenschaften

Nicole Kaiser:

Geplante Wege nach der Promotion. Gründe für den Ausstieg

wissenschaftsorientierter Promovierender aus dem Wissenschaftssystem

Svea Korff:

„Bin ich auf dem richtigen Weg?“ Der Ausstieg aus der Wissenschaft als permanente Option im ereignisgestützten Orientierungsprozess der Postdoc-Phase

Manuela Tischler:

Können Vertrauenserfahrungen den Ausstieg aus der Wissenschaft abwenden? .66

Sigrid Metz-Göckel:

Auf den Spuren der wissenschaftlichen Drop-Outs .76

Hildegard Matthies, Stella Rehbein:

Inkongruenz. Ausstieg aus der Wissenschaft als Folge eines

Mismatch zwischen Feld und Habitus. 


\section{FORUM}

Ulrich Teichler:

Fünf Jahrzehnte des Experimentierens. Hochschulsteuerung und die Gestaltung der Hochschullehrerrolle. Teil 1

Timo Becker:

Kompetenzorientierung Revisited. Eine kritische Betrachtung des

Konzeptes der Kompetenzorientierung in der Hochschullehre 116

Jörg-Peter Pahl, Hannes Ranke:

Forschung an Hochschulen für Angewandte Wissenschaften.

Eine nicht ganz neue Aufgabe?

\section{PUBLIKATIONEN}

Peer Pasternack, Daniel Hechler:

Bibliografie: Wissenschaft \& Hochschulen in Ostdeutschland seit 1945

Autorinnen \& Autoren 174 


\section{Auf den Spuren der wissenschaftlichen Drop-Outs}

\author{
Sigrid Metz-Göckel \\ Dortmund
}

Wissenschaftliche Arbeit hat eine faszinierende und eine problematische Seite. Das Faszinierende an der wissenschaftlichen Arbeit besteht darin, dass die Identifizierung mit dem Inhalt als Lernprozess und persönliche, intellektuelle Befriedigung erlebt werden kann. Nicht umsonst wird das Berufsfeld auch mit dem der Kunst in Verbindung gebracht. So äußert Siri Hustvedt im Gespräch mit dem Neurowissenschaftler Vittorio Gallese: „Was Wissenschaft und Kunst verbindet, ist menschliche Neugier" (Hustvedt/Gallese 2016).

Sandra Beaufaÿs (2015: 40) schreibt mit Bezug auf Bourdieu, von ,seiner' Arbeit fasziniert zu sein, gehöre zum Selbstverständnis kultureller Felder: „Wissenschaftliche Arbeit ist, wie die Kunst, keine Arbeit aus schierer Notwendigkeit, sie ist immer auch eine Form der Selbstaktualisierung". Diese Aussagen spiegeln die verführerische Seite der Wissenschaft wider, entsprechend dem berühmten Ausspruch von Max Weber: „Wem die Leidenschaft fehlt, sollte der Wissenschaft fernbleiben“ (Weber 1975: 12).

Diese ,Idealkonstruktion“ als impliziter wissenschaftlicher Standard trifft in den Universitäten auf eine ganz andere Wirklichkeit. Der universitäre Personalausbau mit seiner Vermehrung der wissenschaftlichen Stellen bei gleichzeitiger Befristung und Stellenteilung hat für die wissenschaftlichen Mitarbeitenden (im Folgenden Mitarbeiter/innen) unstete Beschäftigungsverläufe, Verunsicherung und unberechenbare wissenschaftliche Berufsperspektiven mit sich gebracht, kurz: prekäre Arbeitsbedingungen und fragile Wissenschaftskarrieren (Dörre 2007, Metz-Göckel et al. 2016: 65ff). Für befristet wie unbefristet Beschäftigte ist die Vollzeitbeschäftigung rückläufig. Dieser prekäre Modus der Beschäftigung bildet die problematische Seite der wissenschaftlichen Arbeitsbedingungen. Die größte Personengruppe im wissenschaftlichen Personal befindet sich viele Jahre lang in einer Übergangslage (Klecha/Reimer 2008, Rogge 2015, Metz-Göckel et al. 2016) mit geringen Chancen des 
Verbleibs. ${ }^{1}$ Im Grunde ist das vorab befristete Arbeitsverhältnis, auf das sich die Einzelnen einlassen, bereits eine symbolische Kündigung und ein antizipierter Drop-Out.

Von der Wissenschaft fasziniert und passager in ihr beschäftigt $\mathrm{zu}$ sein, konstituiert ein besonderes Spannungsverhältnis. Profession und Organisation driften auseinander (Schimank 2002) bzw. organisatorische und epistemische Differenzierung bewegen sich gegenläufig (Heintz et al. 2004) - das sind soziologische Deutungen auf der Systemebene für das Auseinanderklaffen von professioneller Idealvorstellung und erlebtem Alltag. Für die individuelle Handlungsebene sind dagegen die subjektiven Konstruktionen in Betracht zu ziehen, die sich als Reaktionen auf die prekären Verhältnisse entwickeln.

Anders als wissenschaftliche Aufstiegskarrieren und -barrieren ist wenig erforscht, welche Alternativen mit dem auslaufenden Arbeitsvertrag wahrgenommen werden, ob auf das Vertragsende ein Abbruch der wissenschaftlichen Beschäftigung, ein Wechsel in einen anderen Arbeitsmarktsektor oder lediglich eine Unterbrechung und dann eine Wiederaufnahme u.a.m. folgt. Dies haben wir quantitativ und qualitativ in dem Projekt „Auf der Suche nach dem verlorenen Nachwuchs. Kurzform Mobile Drop-Outs“ empirisch auf großer Datenbasis genauer untersucht (MetzGöckel et al.: 2016).

\section{Drop-Out Begrifflichkeit für Studium und wissenschaftlich Beschäftigte}

Vorliegende Forschungen beziehen sich im Wesentlichen auf Wissenschaftskarrieren und den wissenschaftlichen Nachwuchs (Konsortium 2013, Kahlert 2013, Kreckel/Zimmermann 2014). Dieser ist aber nur eine Teilmenge des wissenschaftlichen Mittelbaus. Das Forschungsprojekt

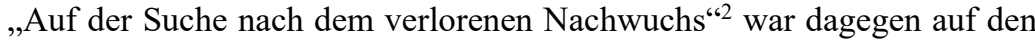
gesamten Mittelbau ausgerichtet. Etwa zwei Drittel des wissenschaftli-

\footnotetext{
1 „Von 2003 bis 2013 ist der akademische Mittelbau an deutschen Universitäten (ohne gleichgestellte Hochschulen) von 120.545 auf 171.037 , d. h. um 50.492 Personen (42\%) angewachsen. Die Zahl der ordentlichen Professuren hingegen ist im gleichen Zeitraum nur um 1.054 (8\%), von 21.129 auf 22.183 gestiegen.“ (Rogge 2015: 693) Das Verhältnis zwischen Professuren und Mittelbau hat sich somit zuungunsten des wissenschaftlichen Nachwuchses verändert.

2 Das Forschungsprojekt „Auf der Suche nach dem verlorenen Nachwuchs. Die mobilen Drop-Outs“ wurde vom BMBF zwischen 2010 und 2013 in der Bekanntmachung ,Frauen an die Spitze' gefördert. Mitarbeiterinnen waren Kirsten Heusgen, Dorothee Koch, Christina Möller, Ramona Schürmann und Petra Selent.
} 
chen Personals waren in der ersten Dekade dieses Jahrhunderts an der Universität beschäftigt, ohne vorzuhaben, langfristig in der Wissenschaft zu bleiben oder eine Professur zu erringen (Janson et al. 2006). Der DropOut aus dem Mittelbau ist demnach der Normalfall.

Die Drop-Out-Begrifflichkeit war lange Zeit mit Studienabbrüchen assoziiert (Heublein et al. 2012, Neugebauer et al. 2019). ${ }^{3}$ Als Abbruch des Studiums ist der studentische Drop-Out kaum exakt zu bestimmen, da eine Wiederaufnahme des Studiums aus einer Studienverlaufsperspektive sehr lange möglich ist. Ebenso kann längere Zeit unklar bleiben, wer sich zum wissenschaftlichen Nachwuchs entwickelt, sodass sich eine Verlaufsperspektive auch für die Situation der Mitarbeiter/ innen anbietet (Schürmann et al. 2016a). Die Ausrichtung auf eine wissenschaftliche Karriere kann im universitären Beschäftigungsverlauf erst entstehen, sie kann auch wieder vergehen und lange diffus bleiben. Dem Drop-Out aus dem universitären Mittelbau kann ebenfalls ein komplexer Prozess vorausgegangen sein, der vor dem endgültigen Ausstieg begonnen hat. Ausstieg aus und Wechsel im Wissenschaftssystem sind somit nicht auf wissenschaftliche Aufstiegskarrieren zu verengen. ${ }^{4}$

\section{Vertragsende und Drop-Out}

Dem Projekt lagen drei eigens hergestellte Datenbestände zugrunde (Selent et al. 2016). Für die Ermittlung der Ausstiegsdaten diente ein Sample von 18 Universitäten (Uni-Daten), die nach Ost-West-Lage, Technische Universität, Volluniversität und Größe zusammengesetzt waren ( $\mathrm{n}=$ 28.534). Diese Universitäten stellten dem Projekt die anonymisierten Personalstandsdaten zur Verfügung. Sie repräsentieren ca. 30 \% des gesamten wissenschaftlichen Mittelbaus an öffentlichen Universitäten. Aus den Daten wurden diejenigen ausgefiltert, deren Vertrag 2009 beendet wurde. Diese wurden als Drop-Out definiert. Zudem wurde 2011 eine Online-

\footnotetext{
${ }^{3}$ In letzter Zeit allerdings wurde sie auch auf strukturierte Promotionsprogramme angewandt (Korff 2015). Wie Abbruchgedanken im Verlauf des Promotionsprozesses entstehen, hat Franz (2018) als sukzessiven Rückzug über kritische Ereignisse rekonstruiert und als symbolischen Tod bezeichnet.

${ }^{4}$ Der Mittelbau selbst ist sehr heterogen bezogen auf die Stellenart (Projekt- oder Haushaltsstelle), die Qualifikation (promoviert oder nicht promoviert (promovierend), die unterschiedlichen Fachrichtungen und Kulturen. Die Beendigung des Beschäftigungsverhältnisses einer bestimmten Universität muss nicht den Ausstieg aus der Wissenschaft bedeuten. Die Arbeit kann ggfs. an einer anderen Universität oder Forschungseinrichtung fortgesetzt werden.
} 
Befragung von Drop-Outs durchgeführt sowie 2012 Interviews mit 20 Ausgestiegenen (7 Frauen und 13 Männer).

Befristete Beschäftigungsverhältnisse haben in der Regel ein vertraglich festgelegtes Ende, daher ist für die Mitarbeiter/innen das Ende ihrer Zeit an der Universität pünktlich vorauszusehen. Allerdings sind mehrere Vertragsverlängerungen die Regel.

Drop-Out, definiert als Ende des Arbeitsvertrags, ist einigermaßen präzise festzustellen. Aus den Universitätsdaten lassen sich die Vertragsbiographien zu einem bestimmten Zeitpunkt konkret rückverfolgen, wie lange die Einzelnen beschäftigt waren und mit welcher Art von Verträgen das Arbeitsverhältnis in 2009 beendet wurde.

Die Auswertung der Drop-Outs ergab in aller Kürze: Knapp ein Fünftel der Mitarbeiter/innen hat 2009 die Universität nach einer Verweildauer von durchschnittlich 4,6 Jahren und 3,6 Verträgen verlassen. Ein Drittel verließ die Universität bereits nach zwei Jahren und weitere $42 \%$ erst nach fünf Jahren, mehr Frauen (19\%) als Männer (17\%). Mehr als die Hälfte (57\%) hatte eine Teilzeitstelle, die Frauen häufiger (66\%) als die Männer (52\%). Fast alle (87\%) waren befristet beschäftigt und etwa gleich häufig promoviert und nicht promoviert (noch promovierend). Die Gründe für den Drop-Out waren:

- Das Ende des Arbeitsvertrags (63\%),

- das Erreichen der Höchstdauer der Beschäftigung nach WissZeitVG $(13 \%)$,

- die vorzeitige Kündigung (24\%) seitens der Universität oder der Mitarbeiter/innen. ${ }^{5}$

Der eingebaute Automatismus der Beendigung durch die befristete Einstellung oder das Erreichen der Höchstdauer der Beschäftigung entlastet die Universitätsverwaltung und macht die weitere Beschäftigung zu einem Problem der Einzelnen. Es sind eher die jüngeren Jahrgänge, ${ }^{6}$ die erste Erfahrungen gesammelt haben und nach knapp fünf Jahren und mehreren kurzfristigen Arbeitsverträgen ausscheiden, darunter sind aber auch vereinzelt Ältere und Habilitierte.

\footnotetext{
${ }^{5}$ Aus den Universitätsdaten ist nicht zu entnehmen, von wem die Kündigung ausging. ${ }^{6} 42 \%$ derjenigen, die 2009 die Universität verlassen haben, gehören zur Gruppe der 26 bis 30-Jährigen, 34 \% zur Gruppe der 31 bis 35-Jährigen (vgl. Metz-Göckel et al. 2016: 72ff.).
} 


\section{Passagere Beschäftigung und wissenschaftliche Identifizierung auf Zeit}

Die strukturell erzeugte personelle Fluktuation fordert die persönliche Mobilität der temporär Beschäftigten heraus, sodass die Verhältnisse einen Modus der Beschäftigung erzeugen, der für die Mitarbeiter/innen ihre Zeit an der Universität zu einer vorübergehenden, zu einer passageren Beschäftigung macht und - um im Wortspiel zu bleiben - lediglich zu einer Passage in ihrem Lebensverlauf. Die Charakterisierung als passagere Beschäftigung akzentuiert das Vorübergehende der Beschäftigung. Der befristete Arbeitsvertrag wird gleichsam zum Ticket, das es ermöglicht, eine bestimmte Strecke im Zug der Wissenschaft mitzufahren. Die Aussteigenden gleichen Reisenden, die eine begrenzte Zeit mitfahren, dann aus- oder umsteigen und/oder den Zielort wechseln. Der Modus der passageren Beschäftigung schließt die prekäre Beschäftigung ein (Selent et al. 2011, Metz-Göckel et al. 2010), ja letztere hat die passagere Beschäftigung erst hervorgebracht. Was aus der institutionellen universitären Perspektive wie ein befristetes Arbeitsverhältnis aussieht, ist im Lebensverlauf der Mitarbeiter/innen eine zeitlich begrenzte Passage.

Für die theoretische Rahmung der subjektiven Konstruktionen, die damit einhergehen, bietet sich das Konzept der ,äußeren“ und ,inneren Karriere' an, das Kahlert (2012) für den Übergang von der Promotion zur Postdoc Phase eingeführt hat. Kahlert hat diese Differenzierung von Edgar Schein (2004) übernommen, der sie im Kontext der Managementforschung entwickelt hat. Für die heterogene Untersuchungsgruppe der Drop-Outs eignet sich das Konzept insofern, als diese wissenschafts- wie berufsorientierte Aussteiger umfasst. Nachwuchswissenschaftler/innen „müssen ihre Karriere planen, und zwar möglichst verschiedene Optionen gleichzeitig, sodass sie sowohl für den Aufstieg als auch für den Ausstieg gerüstet sind" (Kahlert 2012: 63f).

Drop-Outs haben sich gegen die ,äußere Wissenschaftskarriere' mit ihren vorgesehenen Kletterpartien entschieden und ihre wissenschaftliche Identifizierung zur Seite gelegt. Dagegen dürfte die ,innere Karriere', so die Annahme, für sie an Bedeutung gewonnen haben. Die innere Karriere meint nach Schein die ,subjektive Sicht der Person auf die eigene Berufsbiographie, das heißt die Selbsteinschätzung der Entwicklung des Berufslebens" (ebd.: 64). Schein unterscheidet acht Karriereanker, die in kritischen Situationen, z.B. in Übergangssituationen, ihre Wirkungsmacht entfalten, wobei die Orientierung an fachlicher Kompetenz der primäre Karriereanker sei, „der das Selbstkonzept darstellt, das jemand keinesfalls aufzugeben gewillt ist" (ebd.: 65). 
Die von vornherein zeitlich begrenzte wissenschaftliche Tätigkeit lässt sich nur schwer mit der leidenschaftlichen Identifikation in Übereinstimmung bringen, die wissenschaftliche Arbeit kenn- und auch auszeichnet. Äußere und innere Karriere brechen auseinander. Dem gehe ich in den qualitativen Aussagen der Drop-Outs nach.

\section{Drop-Out Erzählungen - ein Blick zurück}

Themen der Interviews ${ }^{7}$ waren, wie die Drop-Outs ihre universitäre und gegenwärtige Beschäftigung erleben bzw. erlebt haben, inwiefern die befristeten Arbeitsverträge ihre subjektiven Erwartungen und wissenschaftliche Identifikation beeinflusst haben, aber auch welche persönlichen Begründungen sie für ihren Ausstieg haben und welchen Belastungen und Erschöpfungssyndromen sie ausgesetzt waren (Metz-Göckel et al. 2016: 121-158).

Die Interviews enthalten viele Erzählungen über Erfahrungen, die zum Ausstieg aus der Universität geführt haben. Dieser wird retrospektiv fast immer gerechtfertigt. Es sind Erinnerungen und Bewertungen im Nachhinein über einen Zeitabschnitt ihres Lebensverlaufs, in den zwischenzeitliche Erfahrungen, nachträgliche Reflexionen und enttäuschte Hoffnungen eingehen. Eine Interviewpartnerin reflektiert dies:

„Die Menschen erzählen das ... in einer anderen Reihenfolge, ja, die haben eine andere Prioritätensetzung, wenn sie etwas erzählen .... Ja, dass einfach die numerische Reihung anders ist als die subjektive nach Wichtigkeit und so" (I 16: 7f).

Die vergegenwärtigte Zeit an der Universität wird mit anderen Berufserfahrungen verglichen und auch re-interpretiert. Einige äußern sich entspannt zu ihrer Universitätszeit und betrachten diese im Nachhinein aus einer erreichten Selbständigkeit heraus, die ihnen neue Optionen eröffnet hat. Andere haben den erlebten Stress ihrer Uni-Zeit hinter sich gelassen und äußern sich sehr kritisch zu dieser Zeit. Andere lassen die Option einer Rückkehr an die Universität offen und wiederum Andere blicken mit einer gewissen Wehmut und Frustration zurück, weil das Umfeld und die Bedingungen sie krankgemacht haben und ihre Wünsche unerfüllt blieben (Schürmann et al. 2016, Metz-Göckel et al. 2016: 169-185).

Einige Äußerungen enthalten Umdeutungen und auch Verzerrungen, z.B. über Lehrdeputate (I 19), Gehaltsstrukturen und Promotionsstandards. Ein inzwischen in der Schule verbeamteter Aussteiger sagt:

\footnotetext{
${ }^{7}$ Zur Kooperation mit den Universitätsverwaltungen siehe Selent et al. (2016: 51-64). Die Interviewten haben sich in der Online-Befragung zu einem Interview bereit erklärt.
} 
„Ich habe bestimmt jetzt fünf Leute gehört, die alle mal eine Diss angefangen hatten und alle haben gesagt: ,Nee, das war mir irgendwie zu doof.' So ein bisschen nach dem Motto: Die noch mit Menschen umgehen können, die gehen dann an eine Schule. Und die halt nicht mit Menschen umgehen können, bleiben an der Uni“" (I 5: 18f).

Auffällig oft ist in den Interviews die Rede davon, dass ihnen Stellen angeboten wurden, selten, dass sie sich darum beworben haben. Ein Interviewpartner berichtet von offenen Grenzen zwischen der universitären und außeruniversitären Beschäftigung:

„Ich kenne sehr viele Leute, die zwischen Wissenschaft und Uni und NichtUni hin und her springen, aber trotzdem in akademischen und forschungsnahen Wirtschaftsbetrieben arbeiten. Und von daher sind die Übergänge sehr viel fließender und auch die Karrierewege sehr viel fließender" (I 6: 30).

Dieses Bild der fließenden Übergänge zwischen dem universitären und außeruniversitären Kontext findet sich mehrfach in den Interviews, aber auch von harten und scharfen Abgrenzungen ist die Rede.

Dass die prekären Beschäftigungsbedingungen die wissenschaftliche Identifikation wider Willen gefährden und sogar zerstören können, berichtet eine Naturwissenschaftlerin:

„Man arbeitet ja nicht für eine Rente ..., das ist das Fatale. Die ganzen Jahre macht man wirklich alles für die Forschung. Und dann trifft man auf diesen rechtsfreien Raum und das war es. Das finde ich ungerecht" (I 7: 18).

Eine promovierte Sozialwissenschaftlerin hat ihre Habilitation abgebrochen. Sie hat als Lehrbeauftragte sechs Jahre unterrichtet. „Ja, das hat mir Spaß gemacht, war nicht gut bezahlt, aber hat Spaß gemacht." Sie habe eine „mordslange Publikationsliste“, sagt sie, weil sie immer geschrieben habe. „Mir macht das ja auch Spaß.“ Auf einer halben wissenschaftlichen Mitarbeiterstelle „habe (ich) halt überwiegend zu Menschenrechtsthemen, Les-bi-schwul-trans-, Frauen, Anti-Gewalt... publiziert, denen war nicht ganz klar, dass das auch meine Themen sind." Sie ist von ihrer Erscheinung her bereits eine ,provokative Andere" und meint, dass ihr Habitus „mit verschiedenen wissenschaftlichen Anforderungen kollidierte“ (I 16: 4).

Trotz der prekären Situation waren einige mit ihrer wissenschaftlichen Arbeit hochgradig identifiziert. Eine Naturwissenschaftlerin, die sich als leidenschaftliche Forscherin bezeichnet, hat sich für den Ausstieg entschieden und sogar überlegt, etwas ganz anderes zu machen:

„Ich war krank, ich war schon im MRT, zwei Mal. Ich glaube, ich hing einfach noch sehr an der Wissenschaft. Also, das war einfach so diese Leidenschaft, die ich aufgeben musste" (I 7:24).

Sie äußert dennoch Verständnis für die Institution: 
„Weil ich krank geworden bin... dann wird man ja auch nutzlos für die Uni.

Irgendwann kann man da ja keine Leistung mehr zeigen" (ebd.).

Auf die Frage, was sie denn krankgemacht habe, antwortet sie:

„Diese Unsicherheit, die Perspektivlosigkeit, der Mangel an Vertrauensver-

hältnis, das ich nie aufbauen konnte zu meiner Chefin (I 7:23).

Ohne die Forschung wäre sie aber „,mega unzufrieden“ geworden. Sie ist jetzt an einer Bundesanstalt unbefristet angestellt, wo sie auch ein wenig Forschung betreiben kann, wie sie sagt.

\section{Die Wechsler/innen und die Aussteiger/innen}

Die Ausgeschiedenen spalten sich zum Zeitpunkt des Interviews grob in Wechsler/innen - Faszinierte und die Aussteiger/innen auf. Es gibt allerdings auch einige Unklare, die auf eine sich bietende Gelegenheit hin die Tätigkeit an der Universität aufgenommen haben und auf weitere Gelegenheiten warten. Ein beim DLR angestellter Physiker sagt: „Wenn man mir eine Fachhochschulprofessur anbietet, weiß ich nicht, ob ich nein sagen würde" (I 20: 27).

Die Wechsler/innen haben die Universität zugunsten einer anderen Universität oder wissenschaftsnahen Institution verlassen. Für einige bringt der Wechsel eine existenzielle Stabilisierung in unbefristeter Anstellung (I 2) und für andere einen Aufstieg in eine Leitungsposition. Für einige bedeutet dieser Wechsel aber auch eine Fortsetzung ihrer bisherigen Situation, z.B. die Beschäftigung in einem (befristeten) DrittmittelProjekt in einer anderen Hochschule oder außeruniversitären wissenschaftsnahen Einrichtung. Einige kehren nach einer (prekären) Phase der Selbständigkeit oder einer außerwissenschaftlichen Berufstätigkeit an die Hochschule zurück, z.B. auf eine Fachhochschulprofessur oder eine Professur auf Zeit (I 13). Dieser Fall ist allerdings in unserem Sample selten.

Die Aussteiger haben das wissenschaftliche Feld verlassen und sind als Lehrer, Ingenieur, Journalist, Freiberufliche u.a.m. in außerwissenschaftlichen Berufsfeldern tätig. Einige schauen mit einer gewissen Wehmut, andere frustriert auf die Zeit an der Universität zurück. Für die meisten dieser Gruppe war die Zeit an der Universität eine Übergangssituation.

Die Aussteiger aus dem Wissenschaftssystem lassen sich noch einmal unterscheiden in Berufszentrierte und Wissenschaftsorientierte.

Die Berufszentrierten waren ohne Verbleibsabsicht im Mittelbau und haben von vornherein nur eine zeitweise universitäre Beschäftigung in- 
tendiert. Für die meisten ist der Ausstieg eine Verbesserung, er kann aber auch (selten) die Fortsetzung einer unsicheren Situation sein (I 16).

Die Wissenschaftsorientierten wären gern in der Wissenschaft geblieben. Ihr Ausstieg hat sich im Verlauf ihrer Zeit an der Universität vorbereitet und ihre anfängliche Karriereambition hat sich durch die Beschäftigungsbedingungen verflüchtigt.

\section{Resümee}

Die Figur temporärer Wissenschaftler/innen ist ein Produkt der strukturellen Beschäftigungsbedingungen, die lediglich eine wissenschaftliche Identifizierung auf Zeit ermöglicht. Die meisten Drop-Outs haben sich der äußeren Karriere mit ihrer Stufenabfolge und dem engen zeitlichen Regime nicht unterworfen. Für diese ist die innere Karriere mit ihren Wertvorstellungen wichtiger. Diese beruht auf ihrer fachlichen Kompetenz, die für sie handlungsleitend ist, so dass sich resümieren lässt: Mit einem abgeschlossenen Studium und einer wenn auch nur kurzen Beschäftigungsdauer an der Universität haben die Drop-Outs ihr Qualifikationsprofil angereichert. Sie sehen ihre universitäre Zeit nicht als verlorene Zeit. In ihrer Wahrnehmung wird der Ausstieg als ,erfolgreicher ${ }^{6}$ Schritt verbucht, der mit der individuellen Lebensplanung übereinstimmte. In seltenen Fällen war er von Enttäuschungen und gar Verletzungen begleitet.

Wenn ca. ein Fünftel der wissenschaftlichen Mitarbeiter/innen jährlich ,ausgewechselt' wird, mag diese personelle Fluktuation aus universitärer Perspektive der Bestenauslese dienen. Aus der Perspektive der Drop-Outs erscheint ihre universitäre Beschäftigung als Passage ihres Lebensverlaufs, die mit einer Besserstellung außerhalb der Universität, in einigen Fällen aber auch mit einer fortgesetzten Unsicherheit verbunden ist. Zu den Ausgestiegenen können auch Faszinierte zählen, die leidenschaftlich bei der Sache waren und enttäuscht wurden. Die anderen Aussteiger unterscheiden sich von ihnen darin, dass ihnen die Bereitschaft fehlt, die wissenschaftliche Arbeit über alles zu stellen.

\section{Literatur}

Beaufaÿs, Sandra (2015): Die Freiheit arbeiten zu dürfen. Akademische Laufbahn und legitime Lebenspraxis, in: Beiträge zur Hochschulforschung 3/2015, S. 40-59.

Dörre, Klaus/Matthias Neis (2008): Forschendes Prekariat? Mögliche Beiträge der Prekarisierungsforschung zur Analyse atypischer Beschäftigungsverhältnisse in der Wissenschaft, in: Stephan Klecha/Wolfgang Krumbein (Hg.): Die Beschäftigungssituation von wissenschaftlichem Nachwuchs, Wiesbaden, S. 127-142. 
Franz, Anja (2018): Symbolischer Tod im wissenschaftlichen Feld, Wiesbaden.

Heintz, Bettina/Martina Merz/Christina Schumacher (2004): Wissenschaft, die Grenzen schafft. Geschlechterkonstellationen im disziplinären Vergleich, Bielefeld.

Hustvedt, Siri/Vittorio Gallese (Iv.) (2016): „Dieses tiefe Begehren, zu begreifen, wer wir sind“. Interview mit Bernd Eberhart und Jessica Sabasch, in: Die Zeit, 29.12.2016, S. 35; URL https://www.zeit.de/2017/01/kunst-wissenschaft-neurowis senschaft-siri-hustvedt-vittorio-gallese (7.2.2020)

Janson, Kerstin/Harald Schomburg/Ulrich Teichler (2006): Wissenschaftliche Wege zur Professur oder ins Abseits? Strukturinformationen zu Arbeitsmarkt und Beschäftigung an Hochschulen in Deutschland und in den USA, Kassel.

Heublein, Ulrich/Johanna Richter/Robert Schmelzer/Dieter Sommer (2012): Die Entwicklung der Schwund- und Studienabbruchquoten an deutschen Hochschulen, Hannover.

Kahlert, Heike (2012): Was kommt nach der Promotion? Karriereorientierungen und pläne des wissenschaftlichen Nachwuchses im Fächer- und Geschlechtervergleich, in: Sandra Beaufaÿs/Anita Engels/Heike Kahlert (Hg.): Einfach Spitze? Neue Geschlechterperspektiven auf Karrieren in der Wissenschaft, Frankfurt/Main.

Kahlert, Heike (2013): Riskante Karrieren. Wissenschaftlicher Nachwuchs im Spiegel der Forschung, Opladen.

Klecha, Stephan/Melanie Reimer (2008): Wissenschaft als besonderer Arbeitsmarkt. Grundtypologie des Umgangs mit unsicherer Beschäftigung beim wissenschaftlichen Personal, in: Stephan Klecha/Wolfgang Krumbein (Hg.), Die Beschäftigungssituation von wissenschaftlichem Nachwuchs, Wiesbaden.

Konsortium Bundesbericht Wissenschaftlicher Nachwuchs (2013): Bundesbericht Wissenschaftlicher Nachwuchs. Statistische Daten und Forschungsbefunde zu Promovierenden und Promovierten in Deutschland, Bielefeld.

Korff, Svea (2015): Lost in Structure. Abbruchgedanken von NachwuchswissenschaftlerInnen in der strukturierten Promotion, Wiesbaden.

Kreckel, Reinhard/Karin Zimmermann (2014): Hasard oder Laufbahn. Akademische Karrierestrukturen im internationalen Vergleich, Leipzig.

Metz-Göckel, Sigrid/Petra Selent/Ramona Schürmann (2010): Integration und Selektion. Dem Dropout von Wissenschaftlerinnen auf der Spur, in: Beiträge zur Hochschulforschung 1/2010, S. 8-35.

Metz-Göckel, Sigrid/Ramona Schürmann/Kirsten Heusgen/Petra Selent (2016): Faszination Wissenschaft und passagere Beschäftigung. Eine Untersuchung zum DropOut aus der Universität, Opladen.

Neugebauer, Martin/Ulrich Heublein/Bettina Hannover (2019): Editorial Studienabbruch, in: Zeitschrift für Erziehungswissenschaft 22, S. 1019-1023.

Rogge, Jan-Christoph (2015): The Winner Takes It All? Die Zukunftsperspektiven des wissenschaftlichen Mittelbaus auf dem akademischen Quasi-Markt, in: Kölner Zeitschrift für Soziologie und Sozialpsychologie, 4/2015, S. 685-707; http://dx.do i.org/10.1007/s11577-015-0341-6

Schein, Edgar (2004): Karriereanker. Handbuch. Die verborgenen Muster in ihrer beruflichen Entwicklung, Darmstadt

Schimank, Uwe (2002): Neue Steuerungssysteme an den Hochschulen. Expertise für die Förderinitiative Science Policy Studies des BMBF, URL www.sciencepolicy studies.de

Schürmann, Ramona/Sigrid Metz-Göckel/Dorothee Koch, Petra Selent (2016): Ressourcen und Beanspruchungserleben von Unibeschäftigten und Drop-Outs, in: 
Sigrid Metz-Göckel, Sigrid/Ramona Schürmann/Kirsten Heusgen/Petra Selent, Faszination Wissenschaft und passagere Beschäftigung. Eine Untersuchung zum Drop-Out aus der Universität, Opladen, S. 121-158.

Schürmann, Ramona/Sigrid Metz-Göckel (2016a): Die Bedeutung der Promotion für den Ausstieg aus oder den Verbleib in der Wissenschaft, in: Sigrid MetzGöckel/Ramona Schürmann/Kirsten Heusgen/Petra Selent: Faszination Wissenschaft und passagere Beschäftigung. Eine Untersuchung zum Drop-Out aus der Universität, Opladen, S. 91-100.

Selent, Petra/Kirsten Heusgen/Dorothee Koch/Ramona Schürmann/Sigrid MetzGöckel (2016): Datenquellen und methodische Ermittlungsschritte zu den DropOuts, in: Sigrid Metz-Göckel/Ramona Schürmann/Kirsten Heusgen/Petra Selent: Faszination Wissenschaft und passagere Beschäftigung. Eine Untersuchung zum Drop-Out aus der Universität, Opladen, S. 51-64.

Selent, Petra/Ramona Schürmann/Sigrid Metz-Göckel (2011): Arbeitsplatz Hochschule. Beschäftigungsbedingungen und Kinderlosigkeit des wissenschaftlichen Personals an Universitäten in Deutschland, in: Ute Klammer/Markus Motz (Hg.), Neue Wege - gleiche Chancen. Expertisen zum Ersten Gleichstellungsbericht der Bundesregierung, Wiesbaden.

Weber, Max (1975): Wissenschaft als Beruf, Berlin. 